\title{
Variation of Polyphenols Composition and Bioactivities of Pomegranate Wines along in Vitro Digestion Process
}

\author{
Li Xuan, Liu Linwei*, Humaira Wasila, Gao Zhongmei, Yuan Tian, Zhao Beita \\ Country College of Food Science and Engineering, Northwest A\&F University, Yangling, Shaanxi, China \\ *Corresponding author: linweiliu8@hotmail.com
}

Received September 09, 2014; Revised October 22, 2014; Accepted October 27, 2014

\begin{abstract}
Six pomegranate wines made from 3 pomegranate varieties were subjected to successive in vitro gastrointestinal digestive system to investigate the effects of pomegranate wine variety and peel addition on the variation of phenols and bioactivities along digestion process. Pomegranate variety contributed more to sugar content, titratable acid level, and alcohol degree, while peel addition mainly accounted for color density and polymer color and phenolics profiles of pomegranate wine. With different wine matrixes and digestive reactions, prime phenolics changed differently along digestion. Different pomegranate wine had different bioactive performances in each digestive stage. Generally, peel wines possessed higher bioactive values than relative aril wines except superoxide anion scavenging ability, and gastric environment were more conducive to DPPH and superoxide anion scavenging, xanthine oxidase inhibiting, but protein denaturation inhibition.
\end{abstract}

Keywords: pomegranate wine, polyphenols, bioactivity, in vitro gastrointestinal digestion

Cite This Article: Li Xuan, Liu Linwei, Humaira Wasila, Gao Zhongmei, Yuan Tian, and Zhao Beita, "Variation of Polyphenols Composition and Bioactivities of Pomegranate Wines along in Vitro Digestion Process." Journal of Food and Nutrition Research, vol. 2, no. 11 (2014): 839-845. doi: 10.12691/jfnr-2-11-13.

\section{Introduction}

Pomegranate has long been regarded as a promising diet source of phytochemicals, including ellagitannin, flavonoids, anthocyanins [1], which possess several health promoting characters, like maintaining redox balance of internal environment, prevention and treatment of cardiovascular disease, diabetes, cancers, Alzheimer`s [2,3]. Pomegranate wine as an emerging beverage not only offers an alternative to employ underused pomegranate fruits [4], but also provide multiple beneficial effects on health management [5]. However, gastro-intestinal bioactivity of pomegranate wine polyphenols are not fully unraveled up to now, despite bioavailability of pomegranate juices had been investigated in vitro by Pérez-Vicente et al. [6].

Manach et al. [7] inferred that bioavailability of polyphenols are highly variable among individuals and many plant polyphenols have limited absorption rates in gastrointestinal tract. However, the unabsorbed polyphenol can be converted to lower molecular weight catabolites by gut enzymes and microorganisms [8], like ellagitannins decomposed into ellagic acid and urolithins by microbial enzymes, which can be partly absorbed or directly play resistance role on colon diseases [2,9,10].

In recent years, the role of dietary polyphenols on preventing upper-gastrointestinal diseases has been widely studied [10]. However, pomegranate wine polyphenol characters and bioactivities variation along gastrointestine are not fully unraveled. In present study, three representative pomegranate varieties were selected and two kinds of juice (juicing use arils; juicing use arils with $50 \%$ peel of the pomegranate) for each variety were prepared for making wines. The wines were then subjected to in vitro successive gastrointestinal digestive system (oral-gastro-intestinal-dialysate) to determine the loss rate of phenolics in four phases of the mimic alimentary tract. And bioactivity of wine digests in each phase was studied by antioxidant activities, protein denaturation inhibition capacity and xanthine oxidase inhibition activity.

\section{Experimental}

\subsection{Material}

Pomegranate fruits (Punica. granatum.L.) of Jingpitian, Sanbaitian and Suanshiliu varieties were procured from the Orchard of Lintong pomegranate base in Shaanxi province of China on harvest time (15th October, 2012) and stored at $4^{\circ} \mathrm{C}$ for a week until processing.

\subsection{Chemicals}

Dry yeast (Saccharomyces cerevisiae) was provided by College of Enology, Northwest A\&F University. HPLC grade phenolic standards were purchased from Vick's biological Inc (Chengdu, Sichuan, China). 2,2-diphenyl-1picrylhydrazyl (DPPH), Tris (hydroxymethyl)- metyl amino methane, pyrogallol, $\alpha$-amylase, pepsin, bovine serum albumin, urea, pancreatin, sodium cholate, and bovine sodium deoxycholate were purchased from Sigma- 
Aldrich Co. (St. Louis, Missouri, USA). Sodium diclofenac, xanthine oxidase, allopurinol were purchased from Tokyo chemical industry Co.LTD (Tokyo, Japan). Dialysis bags (type: P/N 128418, with molecular weight cut-off of $12000 \mathrm{Da}$, thickness 0.005 in, length $15 \mathrm{~cm}$ and width $21 \mathrm{~mm}$ ), were purchased from Sigma-Aldrich Co. (St. Louis, Missouri, USA).

\subsection{Pomegranate Wine Preparation}

Three varieties of pomegranate fruits were cleaned and sterilized by $2 \%$ potassium permanganate solution before juicing. Two kinds of juice were made for each variety: juice from arils (aril juice) and juice from juicing arils with $50 \%$ of mesocarps and epicarps (peel juice). One liter of these fresh juices were immediately subjected to heat treatment $\left(90^{\circ} \mathrm{C}, 30 \mathrm{~s}\right)$, and then blended with pectinase $(0.1 \%, \mathrm{~m} / \mathrm{v})$ and sodium pyrosulfite $(0.0075 \%, \mathrm{~m} / \mathrm{v})$. Each juice was adjusted the soluble solid content to $20 \%(\mathrm{~m} / \mathrm{v})$ by sucrose and $\mathrm{pH} 4.5$ by calcium carbonate before inoculating the activated yeast $(0.02 \%, \mathrm{~m} / \mathrm{v})$. Primary fermentation was conducted at $22^{\circ} \mathrm{C}$ with a speed of 90 r/min for 6 days. Post-fermentation was conducted after sterilization, filtration and bottling, wines were sealed and stored in refrigerator at $4^{\circ} \mathrm{C}$ for 28 days. These pomegranate wines were labeled as Jingpitian aril wine (JPTAW), Jingpitian peel wine (JPTPW), Sanbaitian aril wine (SBTAW), Sanbaitian peel wine (SBTPW), Suanshiliu aril wine (SSLAW), Suanshiliu peel wine (SSLPW). Each wine was prepared in triplicate. Soluble solid content was determined using handhold refractometer. Titratable acid and sugar content were measured according to the official method (AOAC 1984). Color density and polymer color were tested based on the method established by Giusti and Wrolstad [11]. Alcohol degree was determined according to method described by Vigne and Vin [12]. Each measurement was conducted in triplicate.

\subsection{In Vitro Digestion Simulation}

Artificial digestive fluids preparation [13] Saliva was prepared by dissolving $5.208 \mathrm{~g} \mathrm{NaHCO}_{3}, 1.369 \mathrm{~g}$ $\mathrm{K}_{2} \mathrm{HPO}_{4} \cdot 3 \mathrm{H}_{2} \mathrm{O}, 0.877 \mathrm{~g} \mathrm{NaCl}, 0.477 \mathrm{~g} \mathrm{KCl}, 0.441 \mathrm{~g}$ $\mathrm{CaCl}_{2} \cdot 2 \mathrm{H} 2 \mathrm{O}$, $\alpha$-amylase $(200000 \mathrm{U})$, and $0.2 \mathrm{~g}$ urea in distilled water with adjusting $\mathrm{pH} 6.5$ and diluting to $1 \mathrm{~L}$. Gastric fluid was made by dissolving $2.75 \mathrm{~g} \mathrm{NaCl}, 0.27 \mathrm{~g}$ $\mathrm{NaH}_{2} \mathrm{PO}_{4}, 0.82 \mathrm{~g} \mathrm{KCl}, 0.4 \mathrm{~g} \mathrm{CaCl}_{2} \cdot 2 \mathrm{H}_{2} \mathrm{O}, 3.1 \mathrm{~mL} \mathrm{HCl}$, $0.85 \mathrm{~g}$ urea, $34 \mathrm{mg}$ of pepsin, and $1 \mathrm{~g}$ bovine serum albumin in $500 \mathrm{~mL}$ distilled water with adjusting $\mathrm{Ph}$ 1.2. Intestinal fluid was prepared by dissolving $7 \mathrm{~g} \mathrm{NaCl}, 3.38$ g NaHCO $3,0.08$ g KH$_{2} \mathrm{PO}_{4}, 0.564$ g KCl, 0.05 g MgCl$_{2}$, $0.19 \mathrm{~g} \mathrm{CaCl}_{2} \cdot 2 \mathrm{H}_{2} \mathrm{O}, 66.6 \mu \mathrm{L} \mathrm{HCl}, 0.1 \mathrm{~g}$ urea, $120 \mathrm{mg}$ pancreatin, and $1 \mathrm{~g}$ bovine serum albumin in $500 \mathrm{~mL}$ distilled water with adjusting $\mathrm{pH}$ 7.8. Bile was formulated by mixing $0.526 \mathrm{~g} \mathrm{NaCl}, 5.79 \mathrm{~g} \mathrm{NaHCO}_{3}, 0.38 \mathrm{~g} \mathrm{KCl}$, $0.22 \mathrm{~g} \mathrm{CaCl}_{2} \cdot 2 \mathrm{H}_{2} \mathrm{O}, 74 \mu \mathrm{l} \mathrm{HCl}$ and $0.25 \mathrm{~g}$ urea, $0.11 \mathrm{~g}$ sodium cholate, $0.829 \mathrm{~g}$ of sodium deoxycholate and $1.8 \mathrm{~g}$ of bovine serum albumin in $500 \mathrm{~mL}$ distilled water with adjusting $\mathrm{pH}$ 8.0. Dialysate was made by blending equal volume of $\mathrm{NaHCO}_{3}$ solution $(0.5 \mathrm{M})$ and normal saline. Dialysis bag segments were pretreated and soaking in normal saline with one end of each segment sealed with clips.
Successive in vitro gastrointestinal digestion system [14] In vitro digestion were conducted using $50 \mathrm{ml}$ screw top polypropylene tubes, avoiding light and protecting with nitrogen in thermostatic oscillator at $37^{\circ} \mathrm{C}$ with speed of $120 \mathrm{r} / \mathrm{min}$. Firstly, fresh artificial saliva mixed with pomegranate wine to simulate oral digestion for $1 \mathrm{~min}$. Then took out oral sample (O) and frozen at $-20^{\circ} \mathrm{C}$ for further analysis. Thereafter, freshly prepared gastric juice was added into the remained oral digest sample to adjust $\mathrm{pH} 2.0$ and then incubate 1 hour for gastric digestion. And took out gastric sample $(\mathrm{G})$ preserved alike. Immediately afterwards, adjusting the remained gastric digesta to $\mathrm{Ph}$ 6.5 by immersing a prepared dialysis bag filled with $10 \mathrm{ml}$ of bubble free dialysate and incubating for $45 \mathrm{~min}$. After this step, small intestinal digestion was conducted by mixing intestinal fluid and bile (v/v, 4/1) with the liquid outside of dialysis bag, and further incubated for 2 hours. And took intestinal sample (I) and dialysate (D) from outside and inside liquids of the dialysis bag separately and delivered to $-20^{\circ} \mathrm{C}$ for further use. Normal saline instead of the digestive fluids was used as blank along the in vitro digestion, and digest samples of each wine was compared with their blank digest sample to calculate the phenolics loss rate (\%), and dialysable ratio (\%) based on the phenolics concentration of each digest samples revealed by HPLC analysis.

\subsection{Polyphenols Analysis}

Sample pretreatment Original wine and the corresponding digest samples were centrifuged (15000 $\mathrm{rpm}, 10 \mathrm{~min}$ ) at low temperature before subjecting to aqueous two-phase system for polyphenols extraction. Specifically, mixing the sample $(10 \mathrm{~mL})$ with acetone $(6$ $\mathrm{mL}$ ) evenly before adding $3.25 \mathrm{~g}\left(\mathrm{NH}_{4}\right)_{2} \mathrm{SO}_{4}$, then the mixture was treated with ultrasonic wave at $30^{\circ} \mathrm{C}, 40 \mathrm{kHz}$ for $15 \mathrm{~min}$. Until the mixture turned into two phases, supernatant was collected by separating funnel and dried by rotary evaporator. The dried substance was dissolved in $10 \mathrm{~mL}$ methanol, and kept in $-20^{\circ} \mathrm{C}$ for further analysis.

\subsubsection{HPLC Analysis}

HPLC system was equipped with auto-sampler, SPDM20A diode array detector $\left(280 \mathrm{~nm}, 30^{\circ} \mathrm{C}\right)$, and $\mathrm{C} 18$ reversed phase column with $250 \mathrm{X} 21.1 \mathrm{~mm}$ i.d., $5 \mu \mathrm{m}$ particle size and $125 \AA$ pore size. The mobile phase consisted of $2 \%$ acetic acid solution (solvent A) and methanol (solvent B). The flow rate was $0.8 \mathrm{~mL} / \mathrm{min}$. The gradient procedure was optimized as follows: $0-15 \mathrm{~min}, 5-$ 25\% B; 15-25 min, 25\% B; 25-65 min, 25-75\% B; 65-70 min, $15 \% \mathrm{~B} ; 70-75 \mathrm{~min}, 5 \% \mathrm{~B}$. Fifteen micro liters of standard or samples were injected after filtrating through a 0.22 um membrane filter and each sample was measured in triplicate. Individual phenolics was identified and quantified based on the retention time and external calibration curves.

\subsubsection{Antioxidant Activities Determination}

Antioxidant activity was evaluated by three in vitro assays. Total reducing capacity (TRC) was conducted according to method optimized by Ferreira, Baptista et al. [15]. DPPH• free radical scavenging ability (DRS) was determined according to protocol reported by Villano et al. [16]. Superoxide anion scavenging ability (SAS) was 
measured using the method previously reported by Zhang et al. [17]. Distilled water was used as blank and ascorbic acid was used as standard, each sample was tested in triplicate. The results were expressed as mg VcE (ascorbic acid equivalent) $\mathrm{L}^{-1}$ sample respectively.

\subsubsection{Xanthine Oxidase Inhibition Capacity Measurement}

Xanthine oxidase inhibition capacity was assayed following the previously published methods [18] with some modification. In brief, $150 \mathrm{uL}$ of phosphate buffer (14.87 $\mathrm{g} \mathrm{K}_{2} \mathrm{HPO}_{4}, 1.99 \mathrm{~g} \mathrm{KH}_{2} \mathrm{PO}_{4}$ and $0.186 \mathrm{~g}$ EDTA-2Na were mixed and brought to $500 \mathrm{ml}$ with adjusting $\mathrm{Ph} 7.5$ ), $50 \mathrm{uL}$ xanthine oxidase solution $(0.28 \mathrm{U} / \mathrm{mL}$, freshly prepared at $4^{\circ} \mathrm{C}$ ) and $50 \mathrm{uL}$ of properly diluted sample was mixed evenly in a $1.5 \mathrm{~mL}$ centrifuge tube on vortex mixer at $4^{\circ} \mathrm{C}$. Enzyme kinetics was detected at $295 \mathrm{~nm}$ by UV-spectrophotometer immediately after $50 \mathrm{uL}$ of substrate (0.15 mM xanthine dissolved into $\mathrm{pH} 7.5$ phosphate buffer) was added and mixed. Buffer instead of xanthine oxidase solution and solvent instead of relative sample were used as negative control, solvent instead of relative sample was set as sample blank control, and 100 $\mathrm{ug} / \mathrm{mL}$ allopurinol solution instead of sample was used as positive control. Each sample was tested for triplicate. Results were presented as allopurinol equivalent (ug).

\subsubsection{Protein Thermal Denaturation Inhibiting Capacity Measurement}

Based on the previously reported protocols [19] with slight modification, the protein denaturation preventing capacity of wine digests were evaluated. Briefly, the system was built within pH6.3 phosphate buffer $(0.895 \mathrm{~g}$ $\mathrm{Na}_{2} \mathrm{HPO}_{4}, 0.68 \mathrm{~g} \mathrm{KH}_{2} \mathrm{PO}_{4}$ and $3.51 \mathrm{NaCl}$ dissolved in 500 $\mathrm{ml}$ distilled water). Fresh egg albumin was collected and diluted evenly by the phosphate buffer by $(1: 1, \mathrm{v} / \mathrm{v})$, and then fresh egg albumin solution $(0.3 \mathrm{~mL})$ was mixed with appropriate concentration of sample $(2 \mathrm{~mL})$. The mixture was incubated in water bath at $37^{\circ} \mathrm{C}$ for $10 \mathrm{~min}$, thereafter transferred to denaturizing treatment at $70^{\circ} \mathrm{C}$ water bath for $3 \mathrm{~min}$. After cooled down, $2.7 \mathrm{~mL}$ of phosphate buffer was added to uniform the colloidal system, which will be tested the turbidity at $660 \mathrm{~nm}$. Distilled water instead of sample was as blank, and diclofenac sodium as reference; control was set as sample without heat denaturizing treatment. Each sample was measured in duplicate. Results were presented as $\mathrm{mg}$ diclofenac sodium equivalent concentration (DsEC) $\mathrm{mL}^{-1}$.

\subsection{Statistical Analysis}

DPS (Version Rel. 6.55, for Windows, 1997) was used for statistical analysis. The results were reported as the mean and standard deviation of triplicate measurements. Significant differences between means were identified using Duncan's multiple range tests $(\mathrm{p}<0.05$ was considered to be statistically significant).

\section{Results and Discussion}

\subsection{Physicochemical Characteristics of Pomegranate Wine}

Physicochemical properties of the 6 pomegranate wines are listed in Table1. Soluble solid content was in the range of 6.0-8.9 $9^{\circ}$ Brix. Titratable acid content was 4.18-15.67 g citric acid $\mathrm{L}^{-1}$, which falls in the scope of 4.6-20.2 g citric acid $\mathrm{L}^{-1}$ reported in Wonderful, Coupage and Mollar de Elche pomegranate wine [4]. Total sugar content was about $8.48-20.59 \mathrm{~g} \mathrm{~L} \mathrm{~L}^{-1}$ at the end of the primary fermentation, which are almost 4-6 times higher than 0.22-

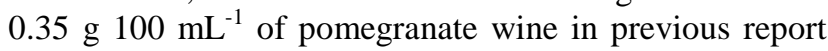
[4]. Polymerized color and color density, reflecting the levels of anthocyanin-tannin complexes, browning index and color contributed by polymerized materials, were affected more by peel addition than by variety difference. Alcohol degree was in the range of $9.67-12.4 \%$, which are $1.3-3.3 \%$ higher than that of three variety pomegranate wines reported by Mena et al. [4] In addition, there were significant differences among varietal wines assayed with regard to fermentation efficiencies.

Polyphenols composition of pomegranate wine is a complex and unique quality character as suggested by Gina Borges et al. [20] In present work, polyphenol profiles of three variety wines had no significant difference, and peel adding noticeably boosted the quantity of phenolics in peel wine. It indicates that fermentation eliminated the discrepancies of the original polyphenol profiles of different pomegranate varieties [21]. These results may contribute new clues for selection of optimal pomegranate materials for fermentation purpose.

Total polyphenol contents of Jingpitian aril wine and peel wine were 57 and $529 \mathrm{mg}$ (gallic acid equivelent) 100 $\mathrm{mL}^{-1}$ respectively, among which total polyphenol content of peel wine is about 1.5 times higher than that of Wonderful (390 mg $100 \mathrm{~mL}^{-1}$ ) and Mollar de Elche (288 $\mathrm{mg} 100 \mathrm{~mL}^{-1}$ ) [4], and 36 kinds of pomegranate beverage (22.58-389.43 mg $100 \mathrm{~mL}^{-1}$ ) [21], whereas total polyphenol content of Jingpitian aril wine ranked at low level in 36 kinds of pomegranate beverages. The main reason is pomegranate juice preparation, in which $50 \%$ of pomegranate peel was fully smashed and blended with juice, resulted in high polyphenol level that largely beat the fermentation lost portion. However, polymerization reactions, enzymes degrading, and oxidation during Jingpitian aril wine elaboration was responsible for its low total polyphenol level.

As can be seen in Table 2, punicalagins were the main polyphenol in pomegranate wine, followed by gallic acid and ellagic acid, which are consistent with other varietal pomegranate wines and pomegranate beverage[21]. Gallic acid contents of both Jingpitian aril wine and peel wine ( 21.55 and $29.74 \mathrm{mg} 100 \mathrm{~mL}^{-1}$ ) are in the similar level with Wonderful, Coupage and Mollar de Elcheare pomegranate wine, (38.1, 20.8 and $15.8 \mathrm{mg} 100 \mathrm{~mL}^{-1}$ respectively) [4]. Different from pomegranate wines reported by Mena et al. [4] and pomegranate beverages reported by Gina Borges et al. [20,22], punicalagin and ellagic acid content of Jingpitian pomegranate wine in this study is the highest among other phenols. It can be explained by different pomegranate variety and juicing method used, as well as primary and post fermentation process that allowed the high level of punicalagin and its derivatives to be formed. In addition, ellagic acid derivatives might be not affected by the fermentation process [23], and a hypothetical balance between degradation and release by hydrolysis of ellagitannins [4] 
could explain the ellagic acid levels in Jingpitian aril and peel wines. Cyanidin 3-glucoside as major individual anthocyanins was detected in this work, which showed excessive low level (7.474 and $4.381 \mathrm{mg} 100 \mathrm{~mL}^{-1}$ ) as compared to the reported three pomegranate wines (8.97$53.04 \mathrm{mg} 100 \mathrm{~mL}^{-1}$ ). It primarily caused by heat treatment, enzymatic treatment before wine processing, and the main fermentation process. Moreover, cyanidin 3-glucoside depletion was showed to be the highest among delphinidin-3,5-diglucoside, cyanidin-3,5-diglucoside and pelargonidin of pomegranate juice [4].

Table 1. Physicochemical characteristics of pomegranate wines

\begin{tabular}{|c|c|c|c|c|c|c|c|c|}
\hline & & $\begin{array}{c}\text { Soluble solid } \\
\left.\text { content ( }{ }^{\circ} \text { Brix }\right)\end{array}$ & $\begin{array}{l}\text { Titratable acid } \\
\text { (g citric acid L- } \\
\text { 1) }\end{array}$ & $\begin{array}{l}\text { Sugar content } \\
\text { (g sucrose L-1) }\end{array}$ & $\begin{array}{l}\text { Sugar-acid } \\
\text { ratio }\end{array}$ & $\begin{array}{l}\text { Color densityf } \\
\text { (A) }\end{array}$ & $\begin{array}{l}\text { Polymer colorg } \\
\text { (A) }\end{array}$ & $\begin{array}{c}\text { Alcohol } \\
\text { degree vol \% }\end{array}$ \\
\hline \multirow{2}{*}{ SBT a } & AW d & $6.10 \pm 0.36 \mathrm{~d}$ & $4.18 \pm 1.02 \mathrm{e}$ & $8.48 \pm 0.97 \mathrm{~d}$ & $2.33 \pm 0.45 \mathrm{c}$ & $4.54 \pm 0.56 \mathrm{e}$ & $3.41 \pm 0.44 \mathrm{e}$ & $12.3 \pm 0.26 \mathrm{a}$ \\
\hline & PW e & $6.00 \pm 0.20 \mathrm{~d}$ & $5.35 \pm 0.96 \mathrm{~d}$ & $16.62 \pm 0.97$ c & $3.27 \pm 0.19$ a & $22.77 \pm 0.75 b$ & $19.11 \pm 0.76 \mathrm{~b}$ & $12.4 \pm 0.30 \mathrm{a}$ \\
\hline \multirow{2}{*}{ JPT b } & AW & $7.16 \pm 0.06 \mathrm{c}$ & $6.92 \pm 0.95 \mathrm{~cd}$ & $18.28 \pm 1.02 \mathrm{~b}$ & $2.64 \pm 0.22 \mathrm{~b}$ & $6.73 \pm 0.70 \mathrm{~d}$ & $7.72 \pm 0.79 \mathrm{~d}$ & $10.8 \pm 0.40 \mathrm{c}$ \\
\hline & PW & $6.00 \pm 0.20 \mathrm{~d}$ & $7.46 \pm 0.97 \mathrm{c}$ & $19.28 \pm 0.96 \mathrm{ab}$ & $2.55 \pm 0.35 b$ & $15.46 \pm 0.86 \mathrm{c}$ & $15.29 \pm 0.88 \mathrm{c}$ & $11.1 \pm 0.31 \mathrm{~b}$ \\
\hline \multirow{2}{*}{ SSLc } & AW & $8.03 \pm 0.15 b$ & $15.67 \pm 0.98 \mathrm{a}$ & $15.02 \pm 1.01 \mathrm{c}$ & $1.18 \pm 0.21 \mathrm{~d}$ & $4.36 \pm 0.57 \mathrm{e}$ & $3.20 \pm 0.60 \mathrm{e}$ & $10.4 \pm 0.44 \mathrm{c}$ \\
\hline & PW & $8.90 \pm 0.36$ a & $13.66 \pm 1.03 \mathrm{~b}$ & $20.59 \pm 0.97 \mathrm{a}$ & $1.44 \pm 0.24 \mathrm{~d}$ & $40.26 \pm 0.65 \mathrm{a}$ & $38.36 \pm 1.04 \mathrm{a}$ & $9.67 \pm 0.25 \mathrm{~d}$ \\
\hline
\end{tabular}

Data were expressed as mean \pm standard deviation $(n=3)$. Different letters represent significant differences $(\mathrm{p}<0.05)$.

a, SBT, (Punica. granatum.L. cv. Sanbaitian); b, JPT, (Punica. granatum.L. cv. Jingpitian); c, SSL, (Punica. granatum.L. cv. Suanshiliu);

$\mathrm{d}$, AW, wine made from aril juice; e, PW, wine made from aril and 1/2 peel mixture juice;

$\mathrm{f}$, color density was presented as A(absorbance) of sample; g,polymer color was presented as A(absorbance) of sample.

$\mathrm{g}$, polymer color was presented as A(absorbance) of sample

\subsection{Polypheols Variation in Digestion Process}

Since wine made by Jingpitian possessed relative intermediate-level of each physiochemical index and higher polyphenols level as compare to the other two varieties, Jingpitian aril wine (JPTAW) and Jingpitian peel wine (JPTPW) was selected for experiments of in vitro gastrointestinal digestion. Polyphenols loss rate of Jingpitian aril wine (JPTAW) and Jingpitian peel wine (JPTPW) in each phase of successive in vitro digestive system is displayed in Table 2.

It can be seen that polyphenol loss rate in oral digestion followed different orders in two different wines. Additionally, gastric condition were more conducive to retain and liberate free phenolics, where lost rate of each phenolics abided by little different sequence in two wines, among which lost rate of punicalagin II, gallic acid, chlorogenic acid, caffeic acid, epicatechine of JPTPW were 2 times higher than those of JPTAW, and lost rate of cyaniding and punicalaginII of JPTPW were about 4 times and 30 times than those of JPTAW respectively.

Intestinal incubation released more punicalagin I (42.574\%) and punicalagin II (34.561\%) from JPTAW, but their lost (74.332\% and 80.962\% respectively) from JPTPW was quite high in simulated intestine. Dialysability of punicalagin I, punicalagin II and gallic acid for JPTAW were 37.33\%, 33.33\% and 33.33\% respectively, which were about 3 times higher than those for JPTPW. Moreover, ellagic acid of JPTAW lost much more in intestine than that of JPTPW. And ellagic acid lost rate of JPTAW intestinal digest were $22.08 \%$ higher than that of gastric; while ellagic acid lost rate of JPTPW intestinal and gastric digests are the same. However ellagic acid dialyzable percentage of JPTAW digest (8.89\%) was less than that of JPTPW digest (33.33\%). Present study were interesting complementary to related research [6] that only ellagitannins hydrolyzing took place. These indicate that isomerisation of punicalagin, precipitation equilibrium with endogenous protein etc. also happened in digestion process.
Anthocyanins vanished in JPTPW intestinal digest, while there was still $21.6 \%$ left in that of JPTAW. Anthocyanins variation along in vitro digestion was comparable with previous study [6,24], that the loss rate was lowest in gastric phase but significantly increased after intestinal digestion, and they were higher dialyzable (25\%) especially for aril wine, revealing that anthocyanins can be directly absorbed in stomach as reported by Manach et al. [7].

Chlorogenic acid increased $26.316 \%$ and $7.46 \%$ in JPTAW and JPTPW intestinal digest respectively. As compare to previous report [25] that apple chlorogenic acid variation in digestion was supposed to be degraded in the period of transition from gastro to intestine, the present result showed chlorogenic acid concentration of JPTAW and JPTPW intestinal digests were 3.33 and 4.50 times than those in gastro respectively. Moreover, chlorogenic acid in JPTPW digests could completely penetrate the dialysis bag, whereas only $66.67 \%$ of that for JPTAW could transit into dialysate. It indicated that substantial degradation and isomerisation of chlorogenic acid in gastro and intestine were greatly affected by wine matrix.

Catechine and epicatechine were depleted in JPTAW intestinal digest, but $1.02 \%$ and $7.08 \%$ were left in that of JPTPW. In consistent with previous study for apple polyphenols in vitro digestion [25], catechin and epicatechine were instable and susceptible to gastric $\mathrm{pH}$ condition. Moreover caffeic acid lost $57.895 \%$ in JPTAW intestinal digest, while completely lost in JPTPW intestinal digest.

Overall high loss rate of individual phenolic was detected in current work, which was in line with previous review researches [2,26]. Different fate of each phenolic in four digestive stages reveals the synergy and rivalry effect among the phenolics. In addition, Jingpitian peel wine subjected to lower phenolics bioaccessibility than JPTAW, since it is more susceptible to complex reactions, such as hydrolyzation, aggregation, coagulation and precipitation [27]. 
Table 2. Dynamic variation of pomegranate wine polyphenols along in vitro digestion process

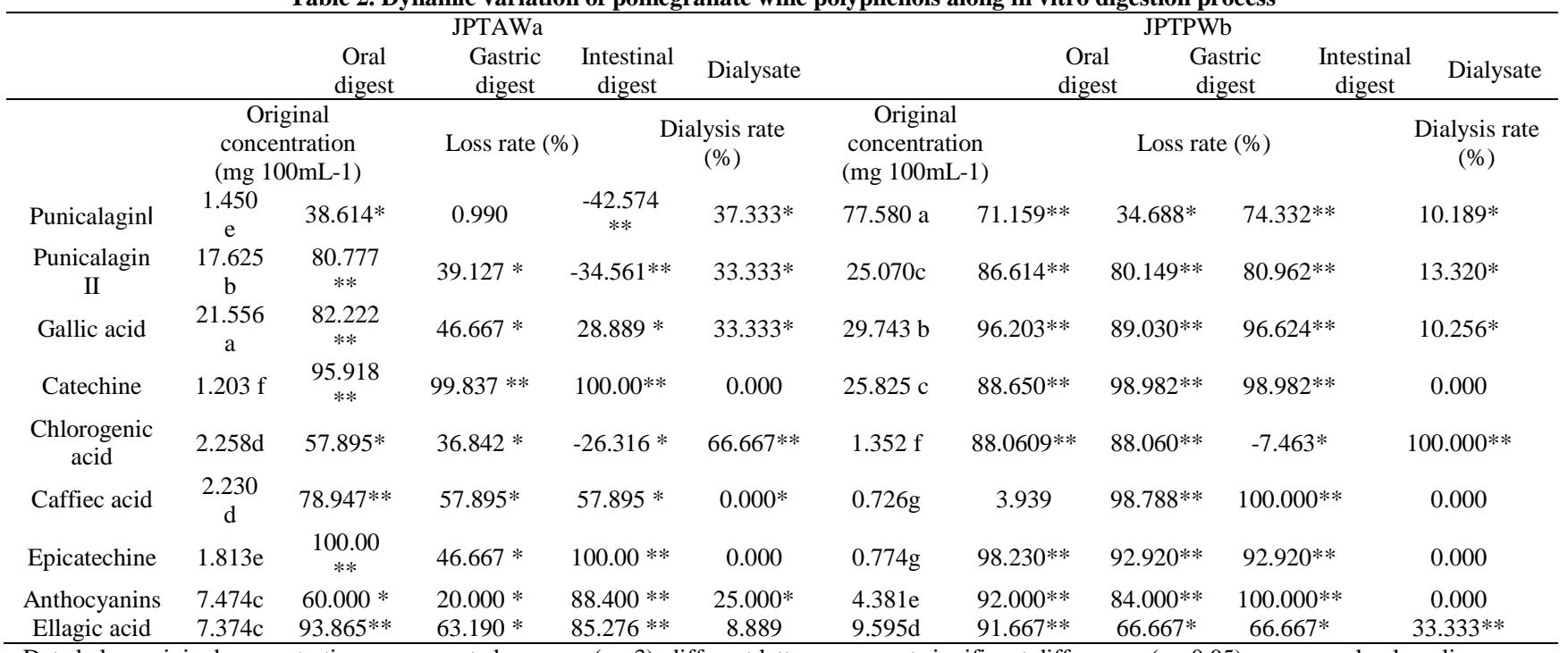

Data below original concentration are presented as mean $(n=3)$, different letters represent significant differences ( $<<0.05)$ among each phenolic compound. Data below loss rate and dialysis rate are presented as mean $(\mathrm{n}=3)$, their significance against control samples are presented as $* * \mathrm{p}<0.01$; $* \mathrm{p}<0.05$.

a, wine made from JPT (Punica. granatum.L. cv. Jingpitia) aril juice; b, wine made from JPT aril with $50 \%$ peels mixture juice.

\subsection{Antioxidant Activities of Pomegranate wine along in Vitro Digestion}

Antioxidant activities variations along gastrointestinal digestion were showed in Figure. The overall tendency of TRC of wine digests displayed stepwise decline from oral digests to dialysates, and all peel wine possessed about 3 times higher TRC than that of aril wine. It caused by polyphenol concentration and the dose-effect relationship indicated in earlier study [25]. DRSC significantly decreased in three peel wines oral digests, indicating that more polyphenols in peel wine than in aril wine precipitated with salivary amylase of saliva. Moreover, DRSC of pomegranate wine gastric digests were remarkably increased (about 30\%-50\% upon oral digests), whereas DRSC of pomegranate wine intestinal digests declined almost $90 \%$ and further reduction was observed in dialysates. These demonstrated that DRSC are not only affected by polyphenol profile, but also acidity feature and buffer condition of digestion environment. SASC of pomegranate peel wines were lower than aril wines along digestion process, which could be verified by our previous work [28]. What's more, aril wine and peel wine gastric digest sample were about 5 times and 3 times of their oral and intestinal digest samples. It means that scavenging capacity of pomegranate wine polyphenols against superoxide anion is enhanced in gastric acidity as compare to oral, intestinal and dialysate condition. Composition and chemical structure of polyphenols played a most key role on antioxidant activity [29], while food matrix would also impact antioxidant activity greatly as pointed by Saura-Calixto et al. [30]. Present work showed antioxidant potential of individual wine in each digestive stage was different despite 3 variety pomegranate wines had similar polyphenol profile. It is speculated that enhancement of antioxidant power of polyphenols in transition from acidic to mild alkaline environment attributed to the deprotonation of the hydroxyl moieties on aromatic rings. Whereas present work showed the protonated pomegranate phenolics had higher potential to eliminate free radicals in acidity environment assessed by DPPH and SAS assays respectively. This may attribute to polyphenols oxidation and other derivatives formed during fermentation, which in need of further investigation.

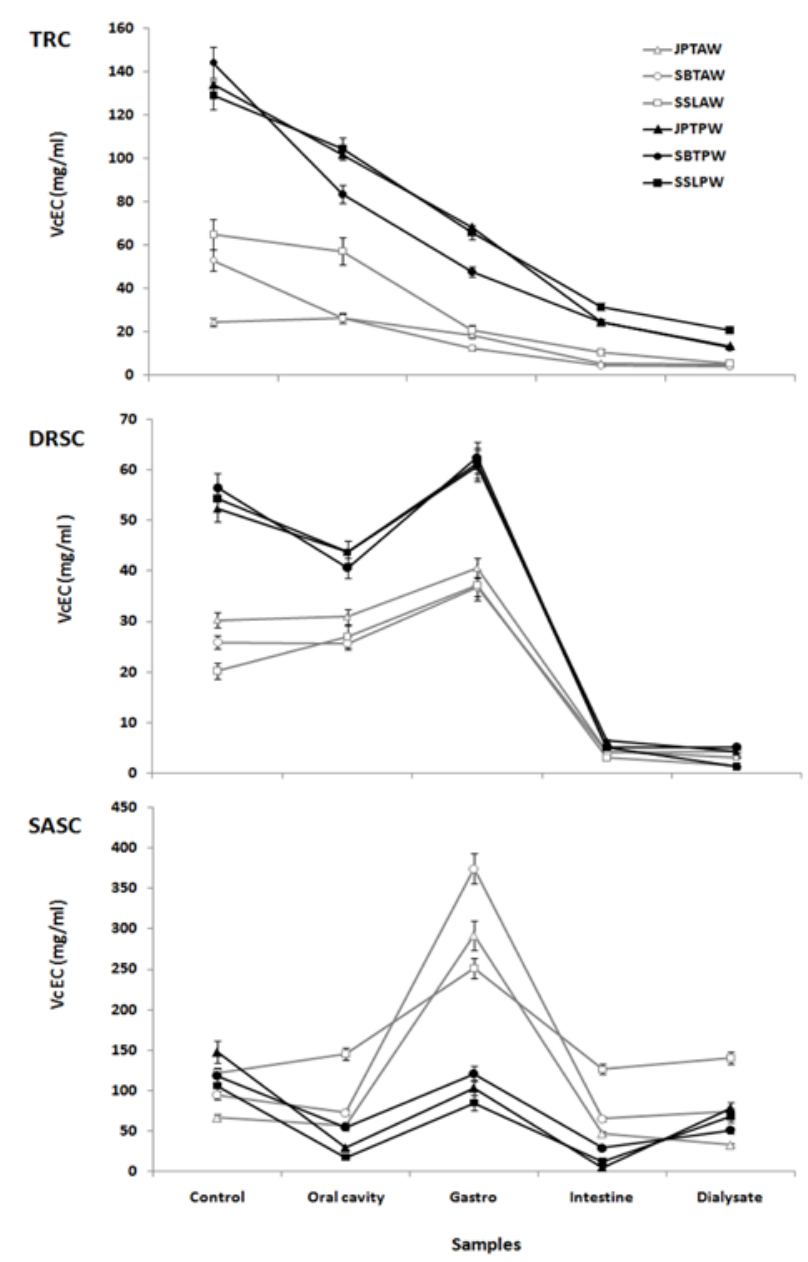

Figure 1. Antioxidant activities of pomegranate wine digestion samples

Data were expressed as ascorbic acid equivalent concentration (VcEC), Vertical bars represent the standard deviation $(n=3)$. TRC, total reducing capacity; DRS, DPPH radical scavenging capacity; SAS, Superoxide anion scavenging capacity 


\subsection{Xanthine Oxidase Inhibition Capacity and Protein Thermal Denaturation Inhibition Capacity of Wine along in Vitro Digestion}

Xanthine oxidase inhibition capacity (XOIC) of six wines digests in in vitro digestion, along with individual phenolic standards were exhibited in Figure 2. The standards all possessed high XOIC. However, all wine digests promoted xanthine oxidase activity except gastric digests. There into, intestinal digests showed stronger xanthine oxidase promotion than oral digests and dialysate. It could be explained that some specific substances in wine facilitated xanthine oxidase activity, while acidic gastric condition might effectively counteract with them. Additionally, there was significant impact of pomegranate variety on XOIC of pomegranate wine in each digestion stage except dialysate.
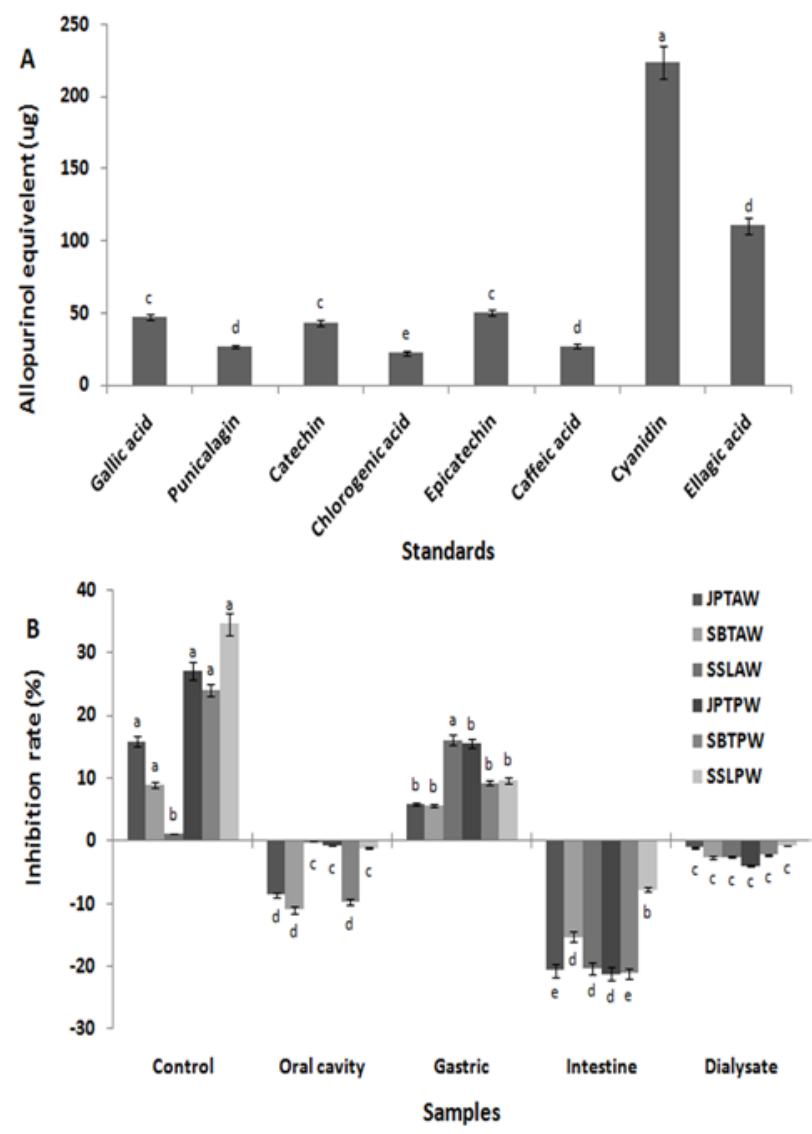

Figure 2. Xanthine oxidase inhibition capacities of pomegranate wine digestion samples

Data were expressed as allopurinol equivalent and inhibition rate (\%). Vertical bars represent the standard deviation $(\mathrm{n}=3)$; Different case letters above the bars represent the significant difference $(p<0.05)$. EC, equivalent concentration.

Protein thermal denaturation inhibition capacity (PDIC) of six pomegranate wine digests is displayed in Figure 3. Oral digests of the 6 wines had higher PDIC than their original wines, which may be caused by the buffer effect of saliva that deduced the alcoholic effect and diluted compounds that is likely to destabilize protein. However, PDIC of six wines gastric digests significantly reduced by 76-90\%, mainly caused by side effect of gastric acidity that shift $\mathrm{pH}$ into isoelectric point for egg albumin. And PDIC of six wines intestinal digests remarkably increased 2.16-12.81 times than that of gastric digests, even though their polyphenol levels are lower than gastric digests. PDIC of six wines dialysate degraded as polyphenol level decreased, which demonstrated that higher quantity and diversity of phenolics have positive effect on protein stable.

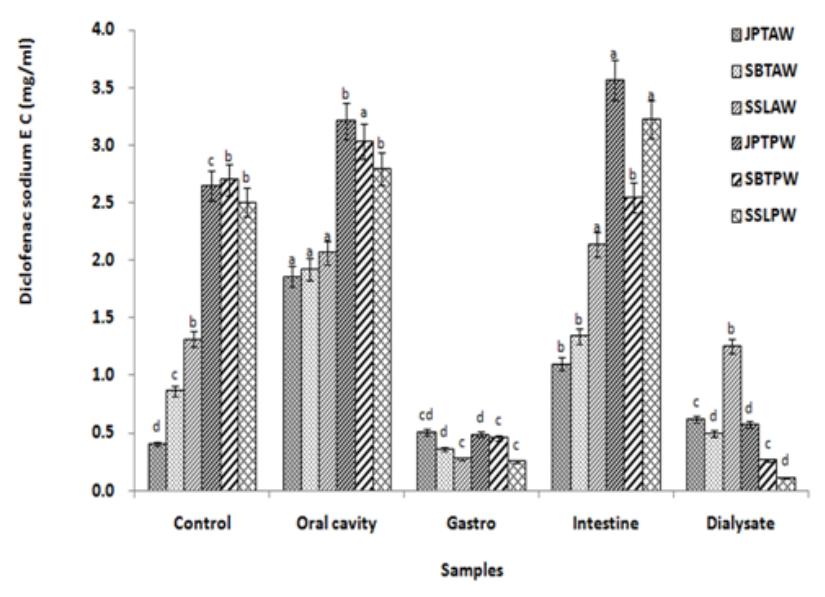

Figure 3. Protein denaturation inhibition capacities of pomegranate wine digestion samples

Data were expressed as diclofenac sodium equivalent concentration. Vertical bars represent the standard deviation $(n=3)$; Different case letters above the bars represent the significant difference $(\mathrm{p}<0.05)$. EC, equivalent concentration.

\section{Conclusion}

To our knowledge this is the first study that focuses on pomegranate wine phenolics profiles and in vitro bioactivities variation along in vitro digestion and their potential bioactivities. Therefore, it is important to demonstrate and share the results to the public for well understanding the effectiveness and health benefits of this emerging drink

\section{Acknowledgement}

Gratitude is expressed to the College of Food Science and Engineering, Northwest A\&F University, for providing facilities and technology support. No conflict of interest exits in the submission of this manuscript, and manuscript is approved by all authors for publication.

\section{References}

[1] Ulrike A Fischer, Reinhold Carle, and Dietmar R Kammerer, "Identification and quantification of phenolic compounds from pomegranate (Punica granatum L.) peel, mesocarp, aril and differently produced juices by HPLC-DAD-ESI/MS n," Food chemistry 127 (2), 807-821 (2011).

[2] JM Landete, "Ellagitannins, ellagic acid and their derived metabolites: a review about source, metabolism, functions and health," Food research international 44 (5), 1150-1160 (2011).

[3] M Viuda-Martos, J Fernández-López, and JA Pérez-Álvarez, "Pomegranate and its many functional components as related to human health: A Review," Comprehensive Reviews in Food Science and Food Safety 9 (6), 635-654 (2010).

[4] Pedro Mena, Amadeo Gironés-Vilaplana, Nuria Martí, and Cristina García-Viguera, "Pomegranate varietal wines: Phytochemical composition and quality parameters," Food Chemistry 133 (1), 108-115 (2012). 
[5] MR Fazeli, S Bahmani, H Jamalifar, and N Samadi, "Effect of probiotication on antioxidant and antibacterial activities of pomegranate juices from sour and sweet cultivars," Natural product research 25 (3), 288-297 (2011).

[6] Antonio Pérez-Vicente, Angel Gil-Izquierdo, and Cristina GarcíaViguera, "In vitro gastrointestinal digestion study of pomegranate juice phenolic compounds, anthocyanins, and vitamin C," Journal of Agricultural and Food Chemistry 50 (8), 2308-2312 (2002).

[7] Claudine Manach, Augustin Scalbert, Christine Morand, Christian Rémésy, and Liliana Jiménez, "Polyphenols: food sources and bioavailability," The American journal of clinical nutrition 79 (5), 727-747 (2004).

[8] Sam Possemiers, Selin Bolca, Willy Verstraete, and Arne Heyerick, "The intestinal microbiome: a separate organ inside the body with the metabolic potential to influence the bioactivity of botanicals," Fitoterapia 82 (1), 53-66 (2011).

[9] Antonio Aguilera-Carbo, Christopher Augur, Lilia A PradoBarragan, Ernesto Favela-Torres, and Cristóbal N Aguilar, "Microbial production of ellagic acid and biodegradation of ellagitannins," Applied microbiology and biotechnology 78 (2), 189-199 (2008).

[10] Mar Larrosa, Antonio González-Sarrías, María J Yáñez-Gascón, María V Selma, María Azorín-Ortuño, Simona Toti, Francisco Tomás-Barberán, Piero Dolara, and Juan Carlos Espín, "Antiinflammatory properties of a pomegranate extract and its metabolite urolithin-A in a colitis rat model and the effect of colon inflammation on phenolic metabolism," The Journal of nutritional biochemistry 21 (8), 717-725 (2010).

[11] M Mónica Giusti and Ronald E Wrolstad, "Characterization and measurement of anthocyanins by UV - visible spectroscopy," Current protocols in food analytical chemistry (2001).

[12] Office International de la Vigne and du Vin, Recueil des méthodes internationales d'analyse des vins et des moûts: édition officielle, juin 1990. (OIV, 1990).

[13] AG Oomen, CJM Rompelberg, MA Bruil, CJG Dobbe, DPKH Pereboom, and AJAM Sips, "Development of an in vitro digestion model for estimating the bioaccessibility of soil contaminants," Archives of Environmental Contamination and Toxicology 44 (3), 0281-0287 (2003).

[14] Sun Jin Hur, Beong Ou Lim, Eric A Decker, and D Julian McClements, "In vitro human digestion models for food applications," Food Chemistry 125 (1), 1-12 (2011).

[15] Isabel CFR Ferreira, Paula Baptista, Miguel Vilas-Boas, and Lillian Barros, "Free-radical scavenging capacity and reducing power of wild edible mushrooms from northeast Portugal: Individual cap and stipe activity," Food Chemistry 100 (4), 15111516 (2007)

[16] D Villano, MS Fernández-Pachón, ML Moyá, AM Troncoso, and MC García-Parrilla, "Radical scavenging ability of polyphenolic compounds towards DPPH free radical," Talanta 71 (1), 230-235 (2007).

[17] Min Zhang, Haixia Chen, Jinlei Li, Ying Pei, and Yi Liang, "Antioxidant properties of tartary buckwheat extracts as affected by different thermal processing methods," LWT-Food Science and Technology 43 (1), 181-185 (2010).

[18] ZS Ferraz Filha, IF Vitolo, LG Fietto, and JA Lombardi, "Xanthine oxidase inhibitory activity of Lychnophora species from Brazil (“Arnica”)," Journal of ethnopharmacology 107 (1), 79-82 (2006).

[19] Sangita Chandra, Protapaditya Dey, and Sanjib Bhattacharya, "Preliminary in vitro assessment of anti-inflammatory property of Mikania scandens flower extract," J Adv Pharm Educ Res 2, 25-31 (2012).

[20] Gina Borges and Alan Crozier, "HPLC-PDA-MS fingerprinting to assess the authenticity of pomegranate beverages," Food chemistry 135 (3), 1863-1867 (2012).

[21] Irine Ginjom, Bruce D’Arcy, Nola Caffin, and Michael Gidley, "Phenolic compound profiles in selected Queensland red wines at all stages of the wine-making process," Food Chemistry 125 (3), 823-834 (2011)

[22] Gina Borges, William Mullen, and Alan Crozier, "Comparison of the polyphenolic composition and antioxidant activity of European commercial fruit juices," Food \& function 1 (1), 73-83 (2010).

[23] MR Pérez-Gregorio, J Regueiro, E Alonso-González, LM Pastrana-Castro, and J Simal-Gándara, "Influence of alcoholic fermentation process on antioxidant activity and phenolic levels from mulberries (Morus nigra L.)," LWT-Food Science and Technology 44 (8), 1793-1801 (2011).

[24] Alan Crozier, Daniele Del Rio, and Michael N Clifford, "Bioavailability of dietary flavonoids and phenolic compounds," Molecular aspects of medicine 31 (6), 446-467 (2010).

[25] Jaouad Bouayed, Lucien Hoffmann, and Torsten Bohn, "Total phenolics, flavonoids, anthocyanins and antioxidant activity following simulated gastro-intestinal digestion and dialysis of apple varieties: Bioaccessibility and potential uptake," Food Chemistry 128 (1), 14-21 (2011).

[26] D Del Rio, LG Costa, MEJ Lean, and A Crozier, "Polyphenols and health: what compounds are involved?," Nutrition, Metabolism and Cardiovascular Diseases 20 (1), 1-6 (2010).

[27] Claudine Manach, Gary Williamson, Christine Morand, Augustin Scalbert, and Christian Rémésy, "Bioavailability and bioefficacy of polyphenols in humans. I. Review of 97 bioavailability studies," The American journal of clinical nutrition 81 (1), 230S-242S (2005).

[28] Humaira Wasila, Xuan Li, Linwei Liu, Imran Ahmad, and Sajjad Ahmad, "Peel Effects on Phenolic Composition, Antioxidant Activity, and Making of Pomegranate Juice and Wine," Journal of food science 78 (8), C1166-C1172 (2013).

[29] Catherine A Rice-Evans, Nicholas J Miller, and George Paganga, "Structure-antioxidant activity relationships of flavonoids and phenolic acids," Free radical biology and medicine 20 (7), 933956 (1996).

[30] Fulgencio Saura-Calixto, José Serrano, and Isabel Goñi, "Intake and bioaccessibility of total polyphenols in a whole diet," Food Chemistry 101 Chemistry 101 (2), 492-501 (2007). 